PROCEEDINGS OF THE

AMERICAN MATHEMATICAL SOCIETY

Volume 136, Number 10, October 2008, Pages 3429-3434

S 0002-9939(08)09426-4

Article electronically published on May 15, 2008

\title{
ON THE UPPER BOUND OF THE MULTIPLICITY CONJECTURE
}

\author{
TONY J. PUTHENPURAKAL
}

(Communicated by Bernd Ulrich)

Dedicated to Juergen Herzog on the occasion of his 65th birthday

\begin{abstract}
Let $A=K\left[X_{1}, \ldots, X_{n}\right]$ and let $I$ be a graded ideal in $A$. We show that the upper bound of the multiplicity conjecture of Herzog, Huneke and Srinivasan holds asymptotically (i.e., for $I^{k}$ and all $k \gg 0$ ) if $I$ belongs to any of the following large classes of ideals:

(1) radical ideals,

(2) monomial ideals with generators in different degrees,

(3) zero-dimensional ideals with generators in different degrees.

Surprisingly, our proof uses local techniques like analyticity, reductions, equimultiplicity and local results like Rees's theorem on multiplicities.
\end{abstract}

\section{INTRODUCTION}

Let $K$ be a field and $A=K\left[X_{1}, \ldots, X_{n}\right]$ be a polynomial ring with standard grading. Let $I$ be a graded ideal of $A$. Let

$$
0 \longrightarrow \bigoplus_{j \in \mathbb{Z}} A(-j)^{\beta_{p, j}(A / I)} \longrightarrow \cdots \longrightarrow \bigoplus_{j \in \mathbb{Z}} A(-j)^{\beta_{1, j}(A / I)} \longrightarrow A \longrightarrow 0
$$

be a minimal graded free resolution of $A / I$. Set $p=\operatorname{projdim} A / I$ and $c=$ height $I$. Consider for $1 \leq i \leq p$ the numbers

$M_{i}(A / I)=\max \left\{j \in \mathbb{Z} \mid \beta_{i, j}(A / I) \neq 0\right\} \& m_{i}(A / I)=\min \left\{j \in \mathbb{Z} \mid \beta_{i, j}(A / I) \neq 0\right\}$.

Let $e(A / I)$ denote the multiplicity of $A / I$. Set

$$
L(I)=\frac{1}{c !} \prod_{i=1}^{c} m_{i}(A / I) \quad \text { and } \quad U(I)=\frac{1}{c !} \prod_{i=1}^{c} M_{i}(A / I) .
$$

The conjecture of Herzog, Huneke and Srinivasan states that

Conjecture 1.1. If $A / I$ is Cohen-Macaulay, then

$$
L(I) \leq e(A / I) \leq U(I) .
$$

If $A / I$ is not Cohen-Macaulay, then in [10] it is conjectured that

Received by the editors January 29, 2007, and, in revised form, September 3, 2007.

2000 Mathematics Subject Classification. Primary 13H15, 13D02; Secondary 13D40, 13A30.

Key words and phrases. Multiplicity conjecture, regularity, reduction, analyticity.

The author thanks the Universtät Duisburg-Essen for its hospitality during November and December of 2006. The author also thanks DFG for financial support, which made this visit possible.

(C)2008 American Mathematical Society Reverts to public domain 28 years from publication 


\section{Conjecture 1.2.}

$$
e(A / I) \leq U(I) .
$$

Both Conjectures 1.1 and 1.2 have been proved for many classes of ideals (see 6, 9, 10, 11, 14, 17, 20, 22, 24]). For extensions of this conjecture see [15, 16, 18, 23, 25. For some new approaches to this problem see [1, 5, 7, 15, 16. Our result is

Theorem 1.3. Let I be a graded ideal. If I belongs to any of the following classes of ideals:

(1) radical ideals,

(2) monomial ideals with generators in different degrees,

(3) zero-dimensional ideals with generators in different degrees, then $e\left(A / I^{k}\right) \leq U\left(I^{k}\right)$ for all $k \gg 0$.

In [11, Theorem 2], the authors show $\lim _{k \rightarrow \infty} e\left(A / I^{k}\right) / U\left(I^{k}\right) \leq 1$. There are examples where $\lim _{k \rightarrow \infty} e\left(A / I^{k}\right) / U\left(I^{k}\right)=1$; for instance see Section 4. In our proof we show that in the class of ideals of Theorem 1.3 we have that the limit on the left hand side is $<1$. The surprising feature of our proof is the use of local techniques like equimultiplicity, reductions analyticity and local theorems like Rees multiplicity theorem (see subsection 2.2).

Overview of the paper. In section 2 we introduce notation and discuss a few preliminary facts that we need. In section 3 we prove Theorem 1.3. In section 4 we give an example of a class of ideals whose members satisfy $\lim _{k \rightarrow \infty} e\left(A / I^{k}\right) / U\left(I^{k}\right)=$ 1.

\section{Preliminaries}

In this section we recall some notions in local algebra. We also discuss asymptotic behavior of regularity of ideals $I^{k}$ for $k \gg 0$. Finally we also recall that the function $k \mapsto e\left(A / I^{k}\right)$ is polynomial in $k$ for $k \gg 0$.

- Some local notions:

Let $(R, \mathfrak{m})$ be a Noetherian local ring of dimension $d$ and residue field $K=R / \mathfrak{m}$ which, for convenience, we assume is infinite. Let $\mathfrak{a}$ be an ideal in $R$. If $M$ is a finitely generated $R$-module, then $\mu(M)$ denotes its minimal number of generators and $\ell(M)$ denotes its length.

2.1. The analytic spread of $\mathfrak{a}$ is the Krull dimension of the fiber-cone $F(\mathfrak{a})=$ $\bigoplus_{n \geq 0} \mathfrak{a}^{n} / \mathfrak{m a} \mathfrak{a}^{n}$. We denote it by $s(\mathfrak{a})$. By [19, p. 150, Th. 1], $s(\mathfrak{a})=\mu(\mathfrak{b})$, where $\mathfrak{b}$ is a (any) minimal reduction of $\mathfrak{a}$. For the definition of reduction and minimal reduction; see [19, p. 146]. It can be shown that height $(\mathfrak{a}) \leq s(\mathfrak{a})$; see [19, p. 151, L. 4]. We say $\mathfrak{a}$ is an equimultiple ideal if height $(\mathfrak{a})=s(\mathfrak{a})$. If $R$ is quasi-unmixed, then $\mathfrak{a}$ is equimultiple if and only if $\operatorname{gr}_{\mathfrak{a}} R=\bigoplus_{n \geq 0} \mathfrak{a}^{n} / \mathfrak{a}^{n+1}$, the associated graded ring of $\mathfrak{a}$, has a homogeneous system of parameters; see [8, 2.6].

2.2. If $\mathfrak{a}$ is $\mathfrak{m}$-primary, then let $e(\mathfrak{a}, R)=$ multiplicity of $R$ with respect to $\mathfrak{a}$; i.e.,

$$
e(\mathfrak{a}, R)=\lim _{n \rightarrow \infty} \frac{d !}{n^{d}} \ell\left(\frac{R}{\mathfrak{a}^{n}}\right) .
$$

Let $\mathfrak{b} \subseteq \mathfrak{a}$ be $\mathfrak{m}$-primary. Clearly $e(\mathfrak{b}, R) \geq e(\mathfrak{a}, R)$. It is easy to see that if $\mathfrak{b}$ is a reduction of $\mathfrak{a}$, then $e(\mathfrak{b}, R)=e(\mathfrak{a}, R)$. A celebrated theorem due to Rees 21] shows that if $R$ is quasi-unmixed and $e(\mathfrak{b}, R)=e(\mathfrak{a}, R)$, then $\mathfrak{b}$ is a reduction of $\mathfrak{a}$.

- Asymptotic behavior of regularity: 
Let $A=K\left[X_{1}, \ldots, X_{n}\right]$. Let $I$ be a graded ideal in $A$ and let $p=\operatorname{projdim} A / I$. Then

$$
\operatorname{reg}(I)=\max \left\{M_{i+1}(A / I)-i \mid i=0, \ldots, p-1\right\}
$$

is the regularity of $I$. Set $\operatorname{reg}_{i}(I)=M_{i+1}(A / I)-i$ for $i=0, \ldots, p-1$.

2.3. In [4, 2.4] and [12, 1] it is shown that $\operatorname{reg}\left(I^{k}\right)=q k+r$ for $k \gg 0$. In [12, 5] it is shown that $I$ has a reduction $J$ such that $\operatorname{reg}_{0}(J)=q$. In particular $J$ is generated in degrees $\leq q$. We call such a reduction a Kodiyalam reduction.

2.4. In [11, 2.1(ii)] it is proved that for $i=0, \ldots, c-1$,

$$
\operatorname{reg}_{i}\left(I^{k}\right)=q k+r_{i} \quad \text { for all } k \gg 0 .
$$

Therefore for $k \gg 0$,

$$
U\left(I^{k}\right)=\frac{1}{c !} \prod_{i=1}^{c} M_{i}\left(A / I^{k}\right)=\frac{q^{c}}{c !} k^{c}+\cdots+\text { lower terms in } k .
$$

- The function $k \mapsto e\left(A / I^{k}\right)$.

2.5. Let $\operatorname{Assh}(I)=\{\mathfrak{P} \in \operatorname{Spec}(A) \mid \mathfrak{P} \supseteq I$ and $\operatorname{dim} A / \mathfrak{P}=\operatorname{dim} A / I\}$. Notice that all $\mathfrak{P} \in \operatorname{Assh}(I)$ are graded ideals. The associativity formula of multiplicity ([2, 4.7.8]) then shows that

$$
e(A / I)=\sum_{\mathfrak{P} \in \operatorname{Assh}(I)} \ell\left(A_{\mathfrak{P}} / I_{\mathfrak{P}}\right) e(A / \mathfrak{P}) .
$$

Since $\operatorname{Assh}\left(I^{k}\right)=\operatorname{Assh}(I)$ for all $k \geq 1$ we have that

$$
e\left(A / I^{k}\right)=\sum_{\mathfrak{P} \in \operatorname{Assh}(I)} \ell\left(A_{\mathfrak{P}} / I_{\mathfrak{P}}^{k}\right) e(A / \mathfrak{P}) .
$$

Recall that $c=$ height $(I)$. Since $k \mapsto \ell\left(A_{\mathfrak{P}} / I_{\mathfrak{P}}^{k}\right)$ is a polynomial function of degree $c$, it follows that $k \mapsto e\left(A / I^{k}\right)$ is a polynomial function of degree $c$. Furthermore, if $E(I)$ is the normalized leading coefficient of this function, then

$$
E(I)=\sum_{\mathfrak{P} \in \operatorname{Assh}(I)} e\left(I_{\mathfrak{P}}, A_{\mathfrak{P}}\right) e(A / \mathfrak{P}) .
$$

Remark 2.6. Let $J \subseteq I$ be a graded ideal. If $J$ is a reduction of $I$, then $J_{\mathfrak{P}}$ is a reduction of $I_{\mathfrak{P}}$ for all primes $\mathfrak{P}$. So $E(J)=E(I)$.

2.7. By subsections 2.4 and 2.5 we get that

$$
\lim _{k \mapsto \infty} \frac{e\left(A / I^{k}\right)}{U\left(I^{k}\right)}=\frac{E(I)}{q^{c}} .
$$

Here $q$ is as in subsection 2.3 .

\section{Proof of Theorem 1.3}

In this section we prove our result. We use [11, Theorem 2], where it is proved that $\lim _{k \rightarrow \infty} e\left(A / I^{k}\right) / U\left(I^{k}\right) \leq 1$. In our proof we show that in the class of ideals of Theorem 1.3 we have that the limit on the left hand side is $<1$. Throughout this section $q$ is as in section 2.3 .

3.1. In [11, section 2] the authors assume $K$ is infinite and then do the following: 
- Let $J$ be a Kodiyalam reduction of $I$ and let $f_{1}, \ldots, f_{c} \in J_{q}$ be $c$-generic $q$-forms. Set $L=\left(f_{1}, \ldots, f_{c}\right)$.

- $e(A / L)=E(L)=q^{c}$.

- $E(I) \leq E(L)$.

The following observation is useful:

Observation 3.2. (1) In subsection 3.1, an ideal $L=\left(f_{1}, \ldots, f_{c}\right)$, where $f_{1}, \ldots, f_{c}$ $\in J_{q}$ is a regular sequence, will do. In fact in [11, section 2] it is chosen generic just to ensure $f_{1}, \ldots, f_{c}$ is a regular sequence.

(2) We may choose $f_{1} \in J_{q}$ to be any non-zero element.

To prove $E(I)<E(L)$ the following remark is useful:

Remark 3.3. $L$ is unmixed. Also height $I=$ height $L=c$. Thus $\operatorname{Assh}(I) \subseteq$ $\operatorname{Assh}(A / L)=\operatorname{Min}(A / L)$, the set of minimum primes of $L$. So to prove $E(I)<$ $E(L)$, it suffices to show that there exists $\mathfrak{P} \in \operatorname{Assh}(I)$ such that $e\left(I_{\mathfrak{P}}, A_{\mathfrak{P}}\right)<$ $e\left(L_{\mathfrak{P}}, A_{\mathfrak{P}}\right)$.

We now give

Proof of Theorem 1.3. We prove that for each class of ideals considered we have $E(I)<E(L)=q^{c}$. We also assume $K$ is infinite. This follows from the usual standard trick in the case when $K$ is finite.

Case 1. I is a radical ideal.

In this case we have the following:

Claim. $E(I)=e(A / I)$.

Let $I=Q_{1} \cap \cdots \cap Q_{s}$ be a minimal irredundant primary decomposition of $I$. Set $\mathfrak{P}_{i}=\sqrt{Q_{i}}$ for $i=1, \ldots, s$. Let $\mathfrak{P} \in \operatorname{Assh}(I)$. Then $\mathfrak{P}=\mathfrak{P}_{i}$ for some $i$. As $I$ is a radical ideal we have $I_{\mathfrak{P}}=\mathfrak{P} A_{\mathfrak{P}}$. Notice $A_{\mathfrak{P}}$ is a regular local ring of dimension $c$. So

$$
\begin{aligned}
e\left(I_{\mathfrak{P}}, A_{\mathfrak{P}}\right) & =e\left(\mathfrak{P} A_{\mathfrak{P}}, A_{\mathfrak{P}}\right)=1, \\
\ell\left(A_{\mathfrak{P}} / I_{\mathfrak{P}}\right) & =\ell\left(A_{\mathfrak{P}} / \mathfrak{P} A_{\mathfrak{P}}\right)=1 .
\end{aligned}
$$

Therefore by (2.1) and (2.3) we get

$$
\begin{aligned}
E(I) & =\sum_{\mathfrak{P} \in \operatorname{Assh}(I)} e\left(I_{\mathfrak{P}}, A_{\mathfrak{P}}\right) e(A / \mathfrak{P})=\sum_{\mathfrak{P} \in \operatorname{Assh}(I)} e(A / \mathfrak{P}), \\
e(A / I) & =\sum_{\mathfrak{P} \in \operatorname{Assh}(I)} \ell\left(A_{\mathfrak{P}} / I_{\mathfrak{P}}\right) e(A / \mathfrak{P})=\sum_{\mathfrak{P} \in \operatorname{Assh}(I)} e(A / \mathfrak{P}) .
\end{aligned}
$$

Thus $E(I)=e(A / I)$.

Set $\mathfrak{m}=\left(X_{1}, \ldots, X_{n}\right)$. Notice that $I_{\mathfrak{m}}$ is a radical ideal of $A_{\mathfrak{m}}$.

Subcase 1. The ideal $I_{\mathfrak{m}}$ is equimultiple. Then by a result due to Cowsik and Nori [3] we have that $I_{\mathfrak{m}}$ is generated by a regular sequence. Since $I$ is graded it follows that $I$ is also generated by a regular sequence. In this case by 9 we have that $e\left(A / I^{k}\right) \leq U\left(I^{k}\right)$ for all $k \geq 1$. 
Subcase 2. $I_{\mathfrak{m}}$ is not equimultiple. Let $L$ be as in subsection 3.1. Then $L$ is not a reduction of $I$. (Otherwise $L_{\mathfrak{m}}$ will be a reduction of $I_{\mathfrak{m}}$ and this will imply that $I_{\mathfrak{m}}$ is equimultiple.)

In particular $L \neq I$. Consider the exact sequence

$$
0 \longrightarrow \frac{I}{L} \longrightarrow \frac{A}{L} \longrightarrow \frac{A}{I} \longrightarrow 0 .
$$

Since $I \neq L$ we have that $(I / L)_{\mathfrak{P}} \neq 0$ for some $\mathfrak{P} \in \operatorname{Ass}(A / L)=\operatorname{Min}(A / L)$. In particular $\operatorname{dim} I / L=\operatorname{dim} A / L=\operatorname{dim} A / I$. It follows that

$$
E(L)=e(A / L)=e(A / I)+e(I / L)>e(A / I)=E(I) .
$$

This implies the result in this case.

Case 2. I is a monomial ideal with generators in different degrees.

Let $\mathfrak{P} \in \operatorname{Assh}(I)$. As $I$ is a monomial ideal, $\mathfrak{P}$ is generated by a subset of the variables [2, 4.4.15]. Say $\mathfrak{P}=\left(X_{i_{1}}, \ldots, X_{i_{s}}\right)$. Let $G(I)=\left\{u_{1}, \ldots, u_{a}\right\}$ be the unique set of minimal monomial generators of $I$. Assume $\operatorname{deg} u_{1}<q$. Set $\alpha=q-\operatorname{deg} u_{1}$. Set $f_{1}=X_{i_{1}}^{\alpha} u_{1}$ and let $L=\left(f_{1}, f_{2}, \ldots, f_{c}\right.$ ) (see Observation $3.2(2)$ ). As $f_{1} \in \mathfrak{P} I_{\mathfrak{P}}$, it follows that $L_{\mathfrak{P}}$ is not a minimal reduction of $I_{\mathfrak{P}}$ [19, Lemma 2]. Therefore by Rees's theorem $e\left(L_{\mathfrak{P}}, A_{\mathfrak{P}}\right)>e\left(I_{\mathfrak{P}}, A_{\mathfrak{P}}\right)$. So by Remark 3.3 we get $E(L)>E(I)$.

Case 3. I is a zero-dimensional ideal with generators in different degrees.

Notice that in this case $\operatorname{Assh}(I)=\left\{\left(X_{1}, \ldots, X_{n}\right)\right\}$. The proof is similar to Case 2.

Remark 3.4. For Cases 2 and 3 in our theorem note that the ideal can never have a pure resolution. Notice also that $\lim _{k \rightarrow \infty}\left\{U\left(I^{k}\right)-e\left(A / I^{k}\right)\right\}=\infty$. This gives further evidence of the "improved" multiplicity conjectures that suggest that Cohen-Macaulay ideals with pure resolutions are the only ones for which the bounds are sharp.

\section{An example}

In [1], the authors state that its easy to construct examples of ideals with $\lim _{k \rightarrow \infty} e\left(A / I^{k}\right) / U\left(I^{k}\right)=1$. For the sake of completeness we give a large class of ideals where $\lim _{k \rightarrow \infty} e\left(A / I^{k}\right) / U\left(I^{k}\right)=1$. The notation will be as in subsection 3 . Set $\mathfrak{m}=\left(X_{1}, \ldots, X_{n}\right)$.

4.1. Let $q \geq 2$ and let $I \subseteq \mathfrak{m}^{q}$ be a zero-dimensional ideal generated by $q$-forms. It is easily verified that $\operatorname{reg}\left(I^{k}\right)=q k+r$ for $k \gg 0$ (use [4, 3.2]). Let $f_{1}, \ldots, f_{n} \in I$ be any regular sequence of $q$-forms. Set $L=\left(f_{1}, \ldots, f_{n}\right)$. Notice that $e\left(L_{\mathfrak{m}}, A_{\mathfrak{m}}\right)=$ $q^{n}=e\left(\mathfrak{m}^{q}, A_{\mathfrak{m}}\right)$. So by a theorem of Rees (see section [2.2), $L_{\mathfrak{m}}$ is a reduction of $\mathfrak{m}^{q} A_{\mathfrak{m}}$. It follows that $L$ is a reduction of $\mathfrak{m}^{q}$. Therefore $L$ is also a reduction of $I$. By Remark 2.6 we get that $E(I)=E(L)=q^{c}$. So by section 2.7 we get $\lim _{k \rightarrow \infty} e\left(A / I^{k}\right) / U\left(I^{k}\right)=1$.

Remark 4.2. We do not know as yet whether the upper bound of the multiplicity conjecture holds asymptotically for all ideals in the class described in subsection 4.1

\section{ACKNOWLEDGMENTS}

It is a pleasure to thank Professor J. Herzog for many discussions regarding this paper. I also thank the referee for a careful reading. 


\section{REFERENCES}

1. M. Boij and J. Söderberg, Graded Betti numbers of Cohen-Macaulay modules and the multiplicity conjecture. Preprint, 2006.

2. W. Bruns and J. Herzog, Cohen-Macaulay rings, Revised edition, Cambridge Studies in Advanced Mathematics 39, Cambridge University Press, Cambridge (1998). MR 1251956 (95h:13020)

3. R.C. Cowsik and M.V. Nori, On the fibres of blowing up. J. Indian Math. Soc. 40, no. 1-4, 217-222 (1976). MR0572990 (58:28011)

4. S.D. Cutkosky, J. Herzog and N.V. Trung, Asymptotic behavior of the Castelnuovo-Mumford regularity, Compositio Math. 118, no. 3, 243-261 (1999). MR1711319 (2000f:13037)

5. C.A. Francisco, New approaches to bounding the multiplicity of an ideal. J. Algebra 299, no. 1, 309-328 (2006). MR2225778 (2007b:13043)

6. L.H. Gold, A degree bound for codimension two lattice ideals. J. Pure Appl. Algebra 182, no. 2-3, 201-207 (2003). MR.1903053 (2004i:13023)

7. L. Gold, H. Schenck and H. Srinivasan, Betti numbers and degree bounds for some linked zero-schemes. J. Pure Appl. Algebra 210, 481-491 (2007). MR2320011

8. U. Grothe, M. Herrmann and U. Orbanz, Graded Cohen-Macaulay rings associated to equimultiple ideals. Math. Z. 186, no. 4, 531-556 (1984). MR744964 (86c:13019)

9. E. Guardo and A. Van Tuyl, Powers of complete intersections: Graded Betti numbers and applications. Ill. J. Math. 49, no. 1, 265-279 (2005). MR2157379 (2006k:13035)

10. J. Herzog and H. Srinivasan, Bounds for multiplicities. Trans. Am. Math. Soc. 350, no. 7, 2879-2902 (1998). MR1458304 (99g:13033)

11. J. Herzog and X. Zheng, Notes on the multiplicity conjecture. Collect. Math. 57, no. 2, 211226 (2006). MR.2223853 (2007a:13029)

12. V. Kodiyalam, Asymptotic behaviour of Castelnuovo-Mumford regularity, Proc. Amer. Math. Soc. 128, no. 2, 407-411 (2000). MR.1621961 (2000c:13027)

13. H. Matsumura, Comutative ring theory. Cambridge Studies in Advanced Math. 8, Cambridge University Press, Cambridge (1986). MR879273 (88h:13001)

14. J. Migliore, U. Nagel and T. Römer, The multiplicity conjecture in low codimensions. Math. Res. Lett. 12, no. 5-6, 731-747 (2005). MR2189234(2006i:13042)

15. J. Migliore, U. Nagel and T. Römer, Extensions of the multiplicity conjecture. Trans. Am. Math. Soc. 360, 2965-2985 (2008). MR 2379783

16. J. Migliore, U. Nagel and F. Zanello, An improved multiplicity conjecture for codimension three Gorenstein algebras. Preprint, 2006.

17. R.M. Miró-Roig, A note on the multiplicity of determinantal ideals. J. Algebra 299, no. 2, 714-724 (2006). MR2228336 (2007a:13030)

18. R.M. Miró-Roig, Betti numbers of determinantal ideals. Preprint, 2007.

19. D.G. Northcott and D. Rees, Reductions of ideals in local rings, Proc. Cambridge. Philos. Soc. 50, 145-158 (1954) MR0059889 (15:596a)

20. I. Novik and E. Swartz, Face ring multiplicity via CM-connectivity sequences. Preprint, 2006.

21. D. Rees, a-transforms of local rings and a theorem on multiplicities of ideals, Proc. Cambridge Philos. Soc. (2) 57, 8-17 (1961). MR0118750(22:9521)

22. T. Römer, Note on bounds for multiplicities. J. Pure Appl. Algebra 195, no. 1, 113-123 (2005). MR2100313 (2005g:13041)

23. T. Römer, Betti numbers and shifts in minimal graded free resolutions. Preprint, 2007.

24. H. Srinivasan, A note on the multiplicities of Gorenstein algebras. J. Algebra 208, no. 2, 425-443 (1998). MR 1655460 (99m:13046)

25. F. Zanello, Improving the bounds in the multiplicity conjecture: the codimension 3 level case. J. Pure Appl. Algebra 209, no. 1, 79-89 (2007). MR2292118(2008a:13034)

Department of Mathematics, Indian Institute of Technology Bombay, Powai, MumBAI, INDIA 400076

E-mail address: tputhen@math.iitb.ac.in 